\title{
Subject Index Vol. 23, 1995
}

Actuarial statistics 133 Acute subdural hematoma 206 Aggressive meningioma 273 Angiography 270 Antiepileptic therapy 311 Apnea 305 Arrested hydrocephalus 20 Arteriovenous malformations,

multiple, cerebral, spinal

166

Back pain 228

Bacterial (mycotic) aneurysm,

antibiotic therapy 97 Basal encephalocele 148 Bone 216,323 Brain stem 279, 323

- glioma 293

water content 246

Cat 246

Cerebral infarction 206

palsy 68, 76, 82 Cerebrospinal fluid 254

- leakage 148

- shunt 86

- - failure, survival 133 Cervical spine 317 Chemotherapy 283

Chiari malformation 260, 323

- II malformation 7 Child abuse 305 Childhood 159 Children 299 Children’s Coma Scale 192

Outcome Scale 192 Chondroid chordoma 159 Chondrosarcoma 159 Chordoma 159

Choroid plexus papilloma 216 Cognitive function 68 Compensated hydrocephalus

20 Compression 323 Computed tomography 171

323 Computerized tomography 93 Congenital abnormalities 86 Cortical mapping 122 Cost analysis 14 Cranial nerves 279 Craniectomy 246 Craniofacial reconstruction 148 Cranioplasty 199 Craniovertebral

junction

anomaly 260 
CT scanning 299 Curly tail 236

Dandy-Walker syndrome 86 Decompression 323 Decompressive craniotomy

192 Delayed brain injury 299 Diabetes mellitus 140 Diffuse axonal injury 305 - brain swelling 305 DNAploidy 283 Durectomy 246 Dynamometry 7

Embolization 166 Encopresis 228 Epidemiology 254 Epilepsy 115, 122 Evoked potentials 192 Experimental hydrocephalus 246 Extradural haematoma 57

Factor VIII 93 Fibrous tract 20 Filum terminale syndrome 228 Fixation device 54 Folicacid 311 Fragile X syndrome 311 Functional magnetic resonance imaging 122

Gait 76

Gastrulation 140 Germ cell tumor(s) 1,219 Germinoma 1 Glasgow Coma Score 64 P-Glycoprotein 283 Grip strength 7

Haemangio-endothelioma 93 Head injury 97, 299 Holoprosencephaly 140 Hydrocephalus 14, 20, 115,

133,254 -, long-term complications

127 -, volumetric analysis 86 Hypertelorism 148

Immunohistology 283 Impact injury 305 Implantation 199 Incontinence 228 Infants 182 Inhibition 68 
Intracranial hematoma 206

- hemorrhage 97

- pressure 192

- monitoring 54

- --, complications 64 Intrathecal baclofen administration 82

Involution 279

Jugular foramen 159

Klinefelter syndrome 219

Lipoma 182

Lipomyelomeningocele 182 Lumbosacral agenesis 140

Magnetic resonance 270

- - imaging 166,171,293

Medulloblastoma 283

Meningeal reaction 171

Meningocele 317

Meningoencephalocele 270

Metaplasia 216

Moyamoya 26

MRA 270

Multidrug resistance 283 Multiple meningiomas 273 Muscle spasticity 82 Myelocystocele 317 Myelography 171 Myelomeningocele 7,311,317, 323

Nasal glioma 148 Neonate 206

Neural tube defects 236, 311 Neurocutaneous syndrome 273 Neuroimaging 122 Neurotrauma, infancy 54 Nonaccidental injury 188 Notochord 159

Optic glioma 1

Paediatric extradural haematoma 57

Pathology 216

Pax-3 236

Perforated demineralized bone matrix 199

Pineal gland 219

Pituitary neoplasm 328

Posterior fossa craniotomy 171

Prognosis 57

Quadriplegia 188 
Regional spread 273 Retinal hemorrhage 305 Reverse-transcriptase PCR 283

Scalp tumour 93 Selective dorsal rhizotomy 68, 76

- rhizotomy 82

Shaken baby syndrome 305

Shaken/impact baby syndrom

192 Shunt 127,246,254

- malfunction 20 Sincipital encephalocele 148 Skull base 159

- defects 199 Slug 236 Spasticity 76

- metastasis 171

- $\quad$ metastasis 171
-
trauma 188 Splotch 236

- $\quad$ trauma 188 Splotch 236

Subdural hemorrhage 305 Subependymal tissue 246 Surgical treatment, children 26 Survey 254 Synostosis 199

Treatment outcome, hydrocephalus 127 Tuberculoma 328 Tuberculous meningitis 328 Tuberous sclerosis 115

Unconscious children, management 64

Valsalva manoeuvre assisted computerized tomography 93

Ventricular enlargement 246

Ventriculoperitoneal shunt 14, 20

Visual processing 68

Waardenburg syndrome 311 